\title{
Reviewers in this issue
}

Dr. Syed Mukarram Ali

Professor of Pathology

Dr. Ataur Rahman Choudhury

Professor of Medicine

Marks Medical College, Dhaka, Bangladesh

Dr. Marium Khatun

Professor of Microbiology

Anwer Khan Modern Medical College, Dhaka, Bangladesh

Dr. S. M. Fazlul Karim

Professor of Biochemistry

Delta Medical College, Dhaka, Bangladesh

\section{Dr. Ferdous Ara}

Professor of Pharmacology \& Therapeutics

Delta Medical College, Dhaka, Bangladesh

Dr. Habibuzzaman Chowdhury

Professor of Forensic Medicine

Dhaka Medical College, Dhaka, Bangladesh

\section{Dr. Rehnuma Jahan}

Associate Consultant of Gynaecology \& Obstetrics

Square Hospital, Dhaka, Bangladesh

\section{Dr. Rezaul Farid Khan}

Associate Professor of Community Medicine

Delta Medical College, Dhaka, Bangladesh 